\title{
Mechanisms by which electroacupuncture-mediated histone acetylation mitigates bone loss in rats with ovariectomy-induced osteoporosis
}

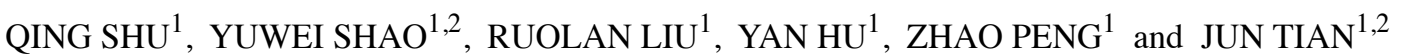 \\ ${ }^{1}$ Department of Rehabilitation, Zhongnan Hospital of Wuhan University; \\ ${ }^{2}$ Second Clinical College, Wuhan University, Wuhan, Hubei 430071, P.R. China
}

Received November 22, 2019; Accepted June 30, 2020

DOI: $10.3892 / \mathrm{mmr} .2020 .11430$

\begin{abstract}
The aim of the present study was to investigate the effectiveness of electroacupuncture (EA) on ovariectomy-induced osteoporotic rats to elucidate potential mechanisms by which EA regulates acetylation of histones in caput femoris. A total of 40 female Sprague-Dawley rats were randomly allocated into four groups: Sham operation, ovariectomy-induced osteoporosis (OVX), EA and 17 $\beta$-estradiol (E2) treatments. After 8 weeks of intervention, the trabecular morphology of each group was measured by micro-computed tomography. Biomarkers of bone metabolism in serum were detected. The protein expression of histone deacetylase 2 (HDAC2), histone H3, Ac-histone H3 and downstream cytokines involved in osteoblast and osteoclast differentiation were detected. The results showed that EA and E2 both prevented bone loss and improved trabecular morphology in OVX rats. EA was found to suppress the protein expression of HDAC2 and promoted the acetylation of histone $\mathrm{H} 3$ compared with the OVX model group. The results indicated that EA promoted the differentiation of osteoblasts, and suppressed that of osteoclasts, thereby improving the trabecular morphology. E2 was shown to regulate the expression of runt-related transcription factor 2 and receptor activator of nuclear factor- $\kappa \mathrm{B}$ ligand without modulating the expression of HDAC2, and therefore diverged mechanistically from EA. Overall, the results of the present study suggested that the mechanisms through which EA improved bone mineral density and trabecular morphology may involve the modulation of histone $\mathrm{H} 3$ acetylation and regulation of osteoblast and osteoclast differentiation.
\end{abstract}

Correspondence to: Professor Jun Tian, Department of Rehabilitation, Zhongnan Hospital of Wuhan University, 169 Donghu Road, Wuchang, Wuhan, Hubei 430071, P.R. China E-mail: tianjun@znhospital.cn

Key words: osteoporosis, electroacupuncture, histone deacetylase 2, osteoblast, osteoclast

\section{Introduction}

Postmenopausal osteoporosis (PMO), which results from estrogen deficiency, is the most common type of osteoporosis and is a systemic skeletal disease that is characterized by low bone mass and micro-architectural deterioration of bone tissues (1). Reduced ovarian production of estrogen after menopause causes an initial phase of rapid bone loss in women, with an annual bone loss rate of $3-5 \%$ for $5-10$ years, which mainly affects the trabecular bone (2). Estrogen replacement therapy is the first line therapy in treating PMO (3); however, side effects such as cardiovascular diseases, cerebrovascular diseases, and invasive breast cancer offsets the beneficial effects on fracture and pain (4). Other treatments, such as bisphosphonates, denosumab and teriparatide, showed efficacy in treating PMO, but extended treatment is challenging for a portion of the population of patients with PMO due to gastrointestinal disturbance or high price $(5,6)$. Acupuncture, which is one of the most popular complementary and alternative therapies in Western countries, has been shown to be effective in improving osteoporosis in several clinical trials (7). Electroacupuncture (EA) is widely used in clinics and is characterized by its precise and reproducible electrical stimulation. EA has shown benefits for rats with ovariectomy-induced osteoporosis by increasing bone mineral density (BMD) (8). The possible mechanisms by which acupuncture has improved BMD may be related to the regulation of osteoblasts and osteoclasts. Runt-related transcription factor 2 (RUNX2) is a key factor in the process of differentiation of mesenchymal stem cells into osteoblasts (9), while receptor activator of nuclear factor- $\kappa \mathrm{B}(\mathrm{RANK})$ ligand (RANKL) has a vital role in the differentiation of macrophages into osteoclasts (10). EA can upregulate the expression of RUNX2 and downregulate the expression of RANK and RANKL, these EA-induced changes activated the formation of osteoblasts and inhibited the formation of osteoclasts (11). Nevertheless, the intrinsic mechanism by which EA regulates these two signaling pathways remains unclear.

Epigenetics is a branch of genetics that addresses the heritable changes in gene expression that does not involve changes in the nucleotide sequence. It mainly includes a large number of histone changes, such as methylation, acetylation and phosphorylation, and these are known to alter gene expression (12). 
Histones are basic proteins that are present in the nucleus of all eukaryotes and bind to DNA. The acetylation of histones, which is regulated by histone deacetylases (HDACs), is an important mechanism that affects chromatin conformation and regulates gene transcription. Histone acetylation usually relaxes chromatin structure, and allows transcription factors and RNA polymerase II to access DNA, which promotes gene transcription (13). In this way, it is associated with the promotion of gene transcription, while histone deacetylation induces compaction of the chromatin and is responsible for blocking gene expression (14). HDAC2, which is widely expressed in bone tissues, activates Akt and thus suppresses FoxO1 transcription, which results in RANKL-induced osteoclastogenesis (15). In addition, HDAC2 has been found to modulate the master transcription factor RUNX2, which is essential in chondrocyte and osteoblast transformation (16). Acupuncture has been shown to regulate the level of histone acetylation by inhibiting the activity of HDAC2 (17). Based on this finding, it was hypothesized that the mechanism by which EA suppresses bone loss may be related to the acetylation modification of histone $\mathrm{H} 3$ by $\mathrm{HDAC} 2$. In the current study four groups were established: The sham operation group (SHAM), ovariectomy-induced osteoporosis group (OVX), EA and $17 \beta$-estradiol (E2) treatment groups, and the mechanism was investigated.

\section{Materials and methods}

Animals models and allocation. A total of 40 female Sprague-Dawley rats (age: 6 month; weight: 315-365 g) were acquired from Vital River Laboratories Co., Ltd., (cat. no. 11400700298971). All rats were placed in a controlled environment at $22 \pm 2^{\circ} \mathrm{C}$ and $50 \pm 10 \%$ relative humidity with a 12-h light/dark cycle in the Experimental Animal Center, Zhongnan Hospital of Wuhan University, with standard food and water provided ad libitum for the duration of the study. After acclimation to the facility for 1 week, all rats were given random ID numbers and randomly allocated to four groups using SPSS 20.0 software. All the rats underwent either SHAM surgery with intact ovaries $(n=10)$ or bilateral ovariectomy (OVX, $\mathrm{n}=30)$. OVX rats were randomly divided into an OVX control group $(n=10)$, EA treatment group $(n=10)$ or E2 treatment group $(n=10)$.

The OVX rats were anesthetized with isoflurane (3-3.5\% for anesthetic induction, 1.8-2.2\% for maintenance of anesthesia), after which both ovaries were removed. The SHAM group underwent surgery where the fat tissue near the ovaries was resected. Following surgery, rats received $9.75 \mathrm{mg} / \mathrm{kg}$ of gentamicin by intramuscular injection each day for 3 days to prevent infection.

Electroacupuncture treatment. Prior to treatment with EA, rats in the EA group were restrained using an instrument made specifically for this experiment to keep them calm (18). Acupoints of Shen Shu (BL23) and Pi Shu (BL20) were subjected to EA by using $0.35 \times 20 \mathrm{~mm}$ acupuncture needles (Global; Suzhou Acupuncture Goods Co., Ltd.) according to current standards (19), briefly: i) BL20 is located at the depression lateral to the lower border of the spinous process of the twelfth thoracic vertebra $(\sim 0.8 \mathrm{~cm}$ in the adult rat, approximately one twentieth of the circumference of the thorax). ii) BL23 is located at the depression lateral to the lower border of the spinous process of the second lumbar vertebra $(\sim 0.8 \mathrm{~cm}$ in the adult rat, approximately one twentieth of the circumference of the thorax).

Bilateral BL20 and BL23 were linked with two electrodes of a Han's Acupoint Nerve Stimulator (HANS LH202H, Neuroscience Research Center, Peking University, Beijing, China). The two acupoints were stimulated with successive waves of $2 \mathrm{~Hz}$, and the intensity varied from $0.5-1 \mathrm{~mA}$, which produce local muscle contractions. Rats in the EA group received EA treatment for 10 min every other day for 8 weeks $(20,21)$, while other groups that did not receive EA were restrained at that time.

E2 intragastric treatment. Rats in the E2 treatment group received E2 intragastric administration with $100 \mu \mathrm{g} / \mathrm{kg}$ E2 $(20 \mu \mathrm{g} / \mathrm{ml})$ at the same time as the EA group underwent treatment for 8 weeks.

Hematoxylin and eosin $(H \& E)$ and tartrate-resistant acid phosphatase (TRACP) staining. Caput femoris were randomly selected from each group and fixed with a $10 \%$ ethylene diamine tetraacetic acid solution $\left(\mathrm{pH} \mathrm{7.4)}\right.$ ) at $4^{\circ} \mathrm{C}$ for 7 weeks. $\mathrm{H} \& \mathrm{E}$ staining was performed on the paraffin sections cut into $5 \mu \mathrm{m}$ sections. Differences in morphology of the H\&E-stained sections of caput femoris were examined, without any morphometric calculation, such as trabecular thickness and trabecular number. TRACP staining was performed with a standard immunohistochemical protocol. Sections were blocked in $0.1 \%$ goat serum (cat. no. AR0009, Wuhan Boster Biological Technology, Ltd.) for $30 \mathrm{~min}$ at room temperature, then incubated at $4^{\circ} \mathrm{C}$ overnight with mouse anti-rat TRACP primary antibody (1:100; cat. no. NBP2-45294; Novus Biologicals, LLC). Finally, a commercial immunohistochemistry kit (cat. no. K5007; Dako; Agilent) including horseradish peroxidase-conjugated anti-mouse secondary antibody and DAB color development kit was used to visualize positive expression, labeling osteoclasts as brown-labelled cells.

Micro-CT bone analysis. Three rats were randomly selected from each group. After abdominal anesthesia with isoflurane and sacrifice by cervical dislocation, the right femur was bluntly dissected to avoid bleeding. All 12 right femurs were imaged on a micro-CT system (SkyScan 1176; Bruker Biospin) according to the manufacturer's protocol to scan the proximal femur with a spatial resolution of $9 \mu \mathrm{m}$ (X-ray source $74 \mathrm{kV}, 338 \mu \mathrm{A} ; 12.59 \mu \mathrm{m}$ filter applied). The distance between each slide was $9 \mu \mathrm{m}$. After a short offset of the first 191 slides from the reference slide, 2,284 continuous slides of CT scan images were included for the trabecular region selection in the proximal femur. The trabecular region of interest was selected in an unbiased analysis using CT-Analyzer software (version 1.15, Bruker) with the suggested order set. The trabecular analysis was performed to quantify BMD and morphometric calculations. All radiographic studies were conducted and quantified blindly with unbiased algorithms and automatic calculations by an independent radiologist. The trabecular metric parameters measured included cortical BMD, trabecular BMD, bone volumetric fraction (BV/TV), trabecular thickness ( $\mathrm{Tb} \mathrm{Th}$ ), 
Table I. Primer sequences

\begin{tabular}{llr}
\hline Primer name & \multicolumn{1}{c}{ Sequence } & Size (bp) \\
\hline Rat $\beta$-actin F & 5'-CACGATGGAGGGGCCGGACTCATC-3' & 240 \\
Rat $\beta$-actin R & 5'-TAAAGACCTCTATGCCAACACAGT-3' & 240 \\
Rat RUNX2 F & 5'-TCAGCGTCCTATCAGTTCCC-3' & 174 \\
Rat RUNX2 R & 5'-ATTCAAAACGGTTGGGGAGC-3' & 174 \\
Rat RANKL F & 5'-CATCGGGTTCCCATAAAGTC-3' & 142 \\
Rat RANKL R & 5'-CTGAAGCAAATGTTGGCGTA-3' & 142
\end{tabular}

F, forward; R, reverse; bp, base pairs; RUNX2, runt-related transcription factor 2; RANKL, receptor activator of nuclear factor- $\kappa \mathrm{B}$ ligand.

trabecular separation (Tb.Sp), and trabecular number (Tb.N). Trabecular bone pattern factor (Tb.Pf), which is an inverse measure of trabecular connectivity, and the structural model index (SMI), which estimates the prevalence of rod-like or plate-like trabecula, were also calculated to assess the trabecular morphology.

Biomarkers of bone metabolism. After 8 weeks of intervention, five rats were selected randomly from each group and anesthetized with isoflurane as aforementioned. Serum was collected by cardiac puncture. The level of serum estradiol(E2) was measured using a commercial enzyme-linked immunosorbent assay (ELISA) kit (cat. no. E-EL-0065c; Elabscience Biotechnology Co., Ltd.,). The levels of serum alkaline phosphatase (ALP) and TRACP 5b were detected by a colorimetric method using a commercial colorimetric assay kit (ALP: cat. no. E-BC-K091, Elabscience Biotechnology Co., Ltd.; TRACP: cat. no. A058, Nanjing Jiancheng Bioengineering Institute Co., Ltd.).

Western blotting. Rats were anesthetized and sacrificed by cervical dislocation after 8 weeks of treatment. The femur was bluntly dissected to avoid bleeding, frozen in liquid nitrogen, and stored at $-80^{\circ} \mathrm{C}$. Caput femoris were cut off and ground into bone powder and then homogenized in radioimmunoprecipitation assay buffer (Beyotime Biotechnology Co., Ltd.). Protein supernatants were quantified using the bicinchoninic acid (BCA) method. Denatured proteins $(30 \mu \mathrm{g})$ were separated on $12 \%$ sodium docecyl sulphate polyacrylamide gels (Beyotime Biotechnology Co., Ltd.,). Polyvinylidene fluoride (PVDF) membranes (Merck KGaA) were blocked in phosphate-buffered saline (PBS) containing $0.1 \% \mathrm{v} / \mathrm{v}$ Tween-20 (TBST) and 5\% w/v skimmed milk for $120 \mathrm{~min}$ at room temperature, followed by overnight incubation at $4^{\circ} \mathrm{C}$ with primary antibodies against HDAC2 $(1: 1,000$; cat. no. 12922-3-ap; ProteinTech Group, Inc.,), histone H3 (1:2,000, cat. no. 17168-1-ap; ProteinTech Group, Inc.,), Ac-histone H3 (1:1,000, cat. no. Bs-3776r; Beijing Biosynthesis Biotechnology Co., Ltd.,), RUNX2 (1:1,000, cat. no. Bs-1134r; Beijing Biosynthesis Biotechnology Co., Ltd.,), and RANKL (1:1,000, cat. no. Bs-0747r; Beijing Biosynthesis Biotechnology Co., Ltd.,), Goat anti-rabbit and goat anti-mouse horseradish peroxidase-conjugated $\operatorname{IgG}(1: 5,000$, cat. nos. BA1056 and BA1051; Wuhan Boster Biological Technology Ltd.,) were used to detect primary antibodies. Membranes were scanned and exposed to photographic film. Optical density was semi-quantified by using Glyco Bandscan software (Version 5.0; ProZyme). Protein expression levels were calculated using the optical density (OD) ratio between the target protein and $\beta$-actin loading control.

Reverse transcription-quantitative polymerase chain reaction $(R T-q P C R)$. Total RNA was extracted from rat caput femoris by using TRIzol ${ }^{\circledR}$ reagent (Invitrogen; Thermo Fisher Scientific, Inc.) and RNA concentrations were determined by the absorbance ratio of $260 / 280 \mathrm{~nm}$. Then, $1 \mu \mathrm{g}$ of the extracted RNA was reverse-transcribed into first-strand complementary DNA (cDNA) using a ReverTra Ace ${ }^{\circledR}$ qPCR RT kit (cat. no. R101-01/02; Vazyme Biotech Co., Ltd.). Gene expression of RUNX2 and RANKL were quantified by using a SYBR-Green Real time PCR Master Mix Plus (Q111-02; Vazyme Biotech Co., Ltd.) following the manufacturer's protocols. Measurements were conducted in triplicate under standard reaction conditions and normalized to $\beta$-actin. Primers were obtained from TsingKe Biological Technology Co., Ltd. All temperature cycling (two initial denaturation steps at $50^{\circ} \mathrm{C}$ for $2 \mathrm{~min}$, then $95^{\circ} \mathrm{C}$ for $10 \mathrm{~min}$, and followed by 40 cycles at $95^{\circ} \mathrm{C} 30 \mathrm{sec}$ and $60^{\circ} \mathrm{C} 30 \mathrm{sec}$ for amplification) and gene amplification were conducted in a QuantStudio ${ }^{\mathrm{TM}} 6$ Real-Time PCR Detection System (Thermo Fisher Scientific, Inc.). The primer sequences used are presented in Table I.

Double-labeled immunofluorescence (IF) staining. Double-labeled IF staining of Ac-histone H3/RUNX2, histone H3/RANKL, RUNX2/ALP and RANKL/TRACP were performed in the $5 \mu \mathrm{m}$ thick paraffin sections of the caput femoris. After antigen retrieval, the sections were blocked in $0.1 \%$ goat serum [dissolved in PBS with $0.02 \%$ Triton $\mathrm{X}-100$ (Beyotime Biotechnology Co., Ltd.) for $1 \mathrm{~h}$ at $37^{\circ} \mathrm{C}$ ] and incubated at $4^{\circ} \mathrm{C}$ overnight with primary antibodies against histone H3 (1:100; mouse; cat. no. bsm-33042 m; BIOSS), Ac-histone-H3 (1:100, mouse, cat. no. bsm-33303 m), RUNX2 (1:100; Rabbit; cat. no. AF5186; Affinity Biosciences) and RANKL(1:100; Rabbit; cat.no.ab62516; Abcam). Then sections were probed with a Cy3-conjugated goat-anti-rabbit secondary antibody (1:50; cat. no. BA1032; Wuhan Boster Biological Technology, Ltd.) or Cy3-conjugated goat-anti-mouse secondary antibody (1:50; cat. no. BA1031; Wuhan Boster Biological Technology, Ltd.) for $1 \mathrm{~h}$ at $37^{\circ} \mathrm{C}$. After blocking in 
goat serum a second time, the samples were incubated at $4^{\circ} \mathrm{C}$ overnight with primary antibodies against RANKL (1:100; Rabbit; cat. no. ab62516; Abcam), RUNX2 (1:100; Rabbit; AF5186; Affinity Biosciences), ALP (1:100; cat. no. sc-36576; mouse; Santa Cruz Biotechnology) and TRACP (1:100; cat. no. NBP2-45294; mouse; Novus Biologicals, LLC) and then with FITC-conjugated goat-anti-rabbit secondary antibody (1:50; cat. no. BA1105; Wuhan Boster Biological Technology, Ltd.) or FITC-conjugated goat-anti-mouse secondary antibody (1:50; cat. no. BA1101; Wuhan Boster Biological Technology, Ltd.) for $1 \mathrm{~h}$ at $37^{\circ} \mathrm{C}$. Nuclei were stained with DAPI and images were acquired using an Olympus BX53 digital fluorescence microscope (Olympus Corporation).

Statistical analysis. Western blotting of the caput femoris in each group were repeated three times and the OD of each target protein was included in the statistical analysis. For IF, three sections were randomly selected from each group for analysis. Optical density analysis was performed on the immunofluorescence images using Image Pro Plus software (version 6.0; Media Cybernetics, Inc.). Three images at x400 magnification were captured for each slice to perform optical density analysis, and the mean density was calculated according to integrated option density (IOD) and area in each microscope view. The Pearson's correlation coefficient (PCC) of the IOD of the two molecules in IF was calculated to confirm the correlation. The difference in gene expression was calculated as the gene expression in the treated group compared with that in the control group, which were calculated using CFX Manager Software version 3.0 (Bio-Rad Laboratories, Inc.). Analysis was performed by blinded biostatisticians using SPSS software version 20.0 (SPSS Statistics IBM Corp). The differences of trabecular parameters, protein bands, optical density in IF and relative gene expression among the four groups were analyzed by one-way ANOVA. To assess the difference between two groups, post-hoc multiple comparisons with the Tukey method were conducted if there were differences among all four groups, the tests were two-sided. $\mathrm{P}<0.05$ was considered to indicate a statistically significant difference.

\section{Results}

EA improves the BMD and trabecular morphology comparably to E2 in OVX rats. As shown in Fig. 1A, in the SHAM group, the caput femoris showed complete structure in the trabecular and cortical bone, a considerable number of trabeculae of normal thickness, and small separation between trabeculae. However, in the OVX model group, the number of abnormal trabeculae and amount of impaired cortical bone were visibly increased and thickness was reduced, with frequent empty bone lacunae. In the EA and E2 treatment groups, the caput femoris showed better trabecular structure than in the OVX model group. The E2 treatment group showed complete structure of cortical bone, which was impaired in the EA treatment group. As shown in Fig. 1B, fewer TRACP-stained osteoclasts were found in the SHAM group compared with the OVX group, while there were less osteoclasts in the EA and E2 treatment groups than in the OVX group.
The caput femoris evaluated with micro-CT are shown in Fig. 1C. After 8 weeks of intervention, there was no notable loss of cortical bone from the sagittal mid-section views of caput femoris after OVX. Compared with the SHAM group, OVX rats showed a significant decrease in trabecular bone BMD $(\mathrm{P}<0.01)$, while EA and E2 both spared this trabecular bone mineral loss after OVX. In the OVX group, BV/TV, Tb.Th and Tb.N were significantly lower, whereas Tb.Sp, Tb.Pf and SMI were significantly higher compared with those in SHAM group. Compared with the OVX model group, both EA and E2 treatment groups increased the BV/TV and Tb.N significantly, but did not alter the Tb.Th in caput femoris. The Tb.Pf and SMI were decreased after treatment with EA or E2 (Fig. 1D). All the results supported the hypothesis that EA mitigated the bone loss from ovariectomy-induced osteoporosis in rats through the regulation of the trabecular morphology, on par with E2 treatment.

EA improves biomarkers of bone metabolism comparably with E2 in OVX rats. As shown in Fig. 2A, serum levels of TRACP 5b, which is indicative of the activity of bone resorption, were increased after OVX $(\mathrm{P}<0.01)$. After 8 weeks of treatment, TRACP 5b levels in the EA and E2 treatment groups were decreased, and treatment with E2 by intragastric administration was shown to be significantly more effective than treatment with EA. Levels of ALP and E2 were decreased after OVX $(\mathrm{P}<0.01)$. After 8 weeks of treatment, serum ALP and E2 in the EA and E2 treatment groups were increased, and no significant differences were detected between the EA and E2 treatment groups (Fig. 2B and C).

EA affects the expression of RUNX2 and RANKL by regulating the level of histone acetylation. As shown in Fig. 3, following from OVX, HDAC2 protein expression levels in caput femoris were upregulated, and this upregulation was suppressed after treatment with EA (Fig. 3B). Rats that received treatment with E2 intragastric administration did not show changes in HDAC2 as compared with the OVX model group. Protein expression of histone $\mathrm{H} 3$ showed a similar profile as HDAC2, and was more highly expressed in the OVX model and E2 treatment groups and less expressed in the SHAM group and EA treatment group (Fig. 3C). By contrast, Ac-histone H3 showed a higher expression in the SHAM group and lower expression in the OVX model group. Ac-histone H3 levels were restored following treatment with EA and were not significantly different between the OVX model and E2 treatment groups (Fig. 3D), this indicated that EA can mitigate OVX-suppressed histone $\mathrm{H} 3$ acetylation, while E2 has no such effect. RUNX2 was highly expressed in the SHAM group compared with the OVX model group. Compared with the OVX group, protein expression of RUNX2 was upregulated by both the EA and E2 treatment (Fig. 3E). RANKL was minimally expressed in the SHAM group in comparison with the OVX group. Compared with the OVX group, RANKL protein expression was downregulated in both the EA and E2 groups, and RANKL protein expression after E2 treatment was lower than that after EA treatment (Fig. 3F).

EA affects the gene expression of RUNX2 and RANKL. As shown in Fig. 3G and H, the RNA expression of RUNX2 
A

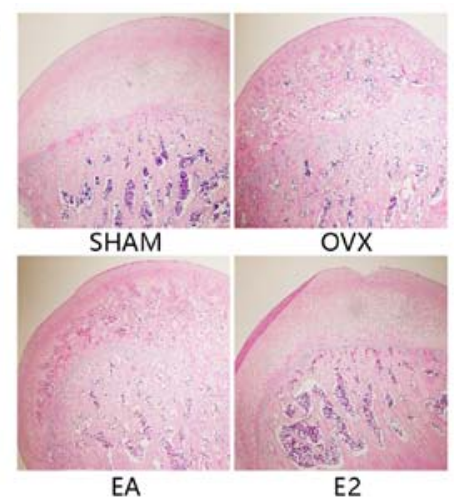

B

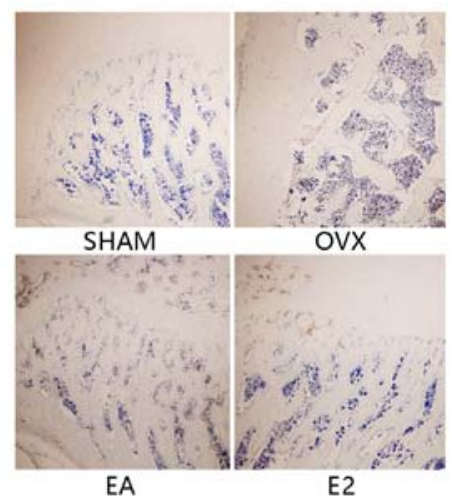

C

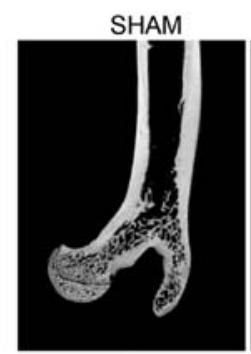

ovX
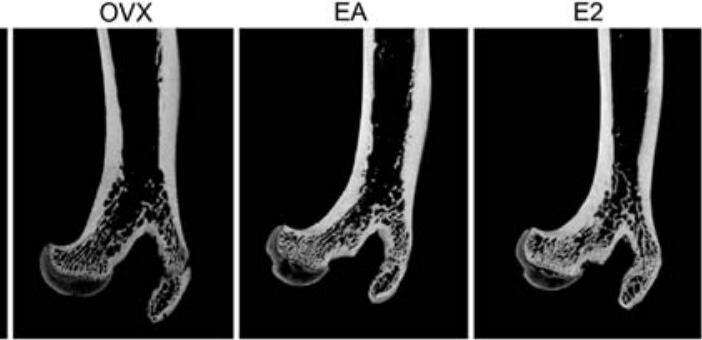

D
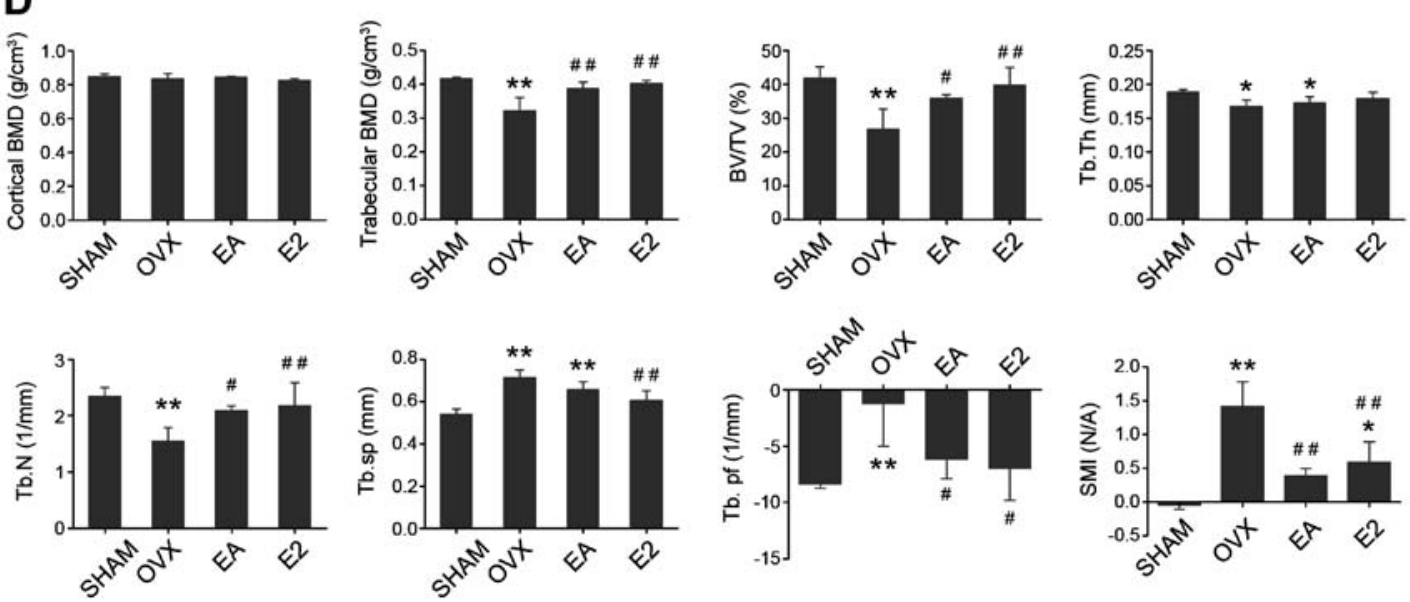

Figure 1. Representative light micrographs of H\&E and TRACP-stained sections of caput femoris and comparison of micro-CT and trabecular metric parameters of caput femoris. (A) Representative images of H\&E-stained sections of caput femoris in SHAM group, OVX model group, EA and E2 treatment groups. (B) Representative images of TRACP staining of caput femoris in SHAM group, OVX model group, EA and E2 treatment groups. (C) Representative images of micro-CT of caput femoris in SHAM group, OVX model group, EA and E2 treatment groups. (D) Trabecular metric parameters of caput femoris in SHAM group, OVX model group, EA and E2 treatment groups. ${ }^{*} \mathrm{P}<0.05,{ }^{* *} \mathrm{P}<0.01$, vs. SHAM; ${ }^{\#} \mathrm{P}<0.05,{ }^{\# \#} \mathrm{P}<0.01$, vs. OVX. SHAM, group given sham surgery leaving the ovaries intact; OVX, bilateral ovariectomy model group; EA, electroacupuncture treatment group; E2, 17ß-estradiol treatment control group; BMD, bone mineral density; BV/TV, bone volumetric fraction; Tb.Th, trabecular thickness; Tb.N, trabecular number; Tb.sp, trabecular separation; Tb.pf, trabecular bone pattern factor; SMI, structural model index.

A

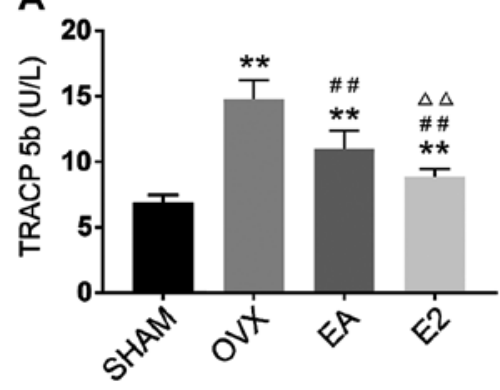

B

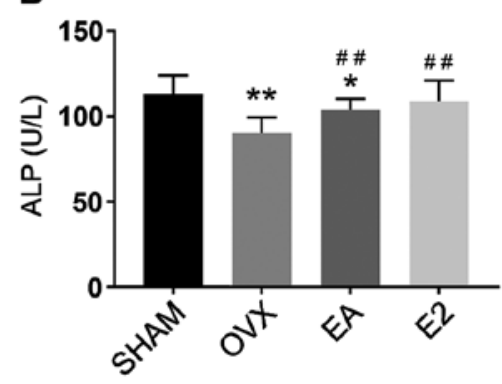

C

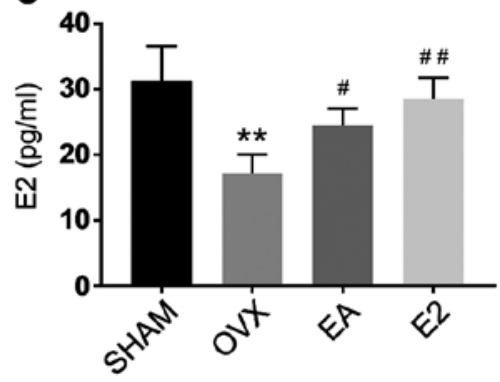

Figure 2. Comparison of bone metabolism biomarkers. (A) Serum TRACP levels in the SHAM, OVX model, EA and E2 treatment groups (B) Serum ALP levels in the four groups. (C) E2 level of serum by colorimetric method in the four groups ${ }^{*} \mathrm{P}<0.05,{ }^{* *} \mathrm{P}<0.01 \mathrm{vs}$. SHAM; ${ }^{\#} \mathrm{P}<0.05,{ }^{\# \#} \mathrm{P}<0.01 \mathrm{vs}$. OVX; ${ }^{\Delta \Delta} \mathrm{P}<0.01$ vs. EA. SHAM, group given sham surgery leaving the ovaries intact; OVX, bilateral ovariectomy model group; EA, electroacupuncture treatment group; E2, $17 \beta$-estradiol treatment control group; TRACP, tartrate-resistant acid phosphatase; ALP, alkaline phosphatase. 
A

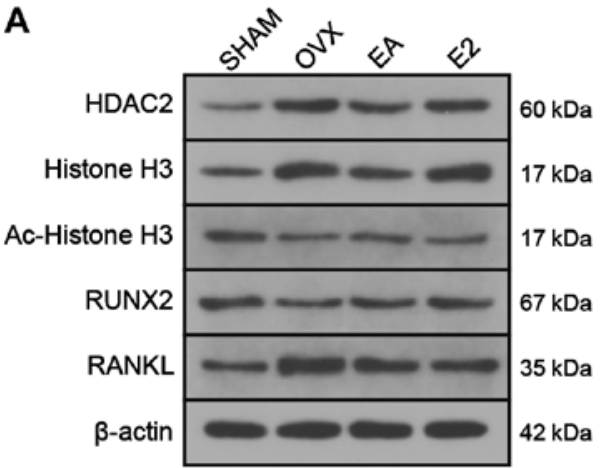

D

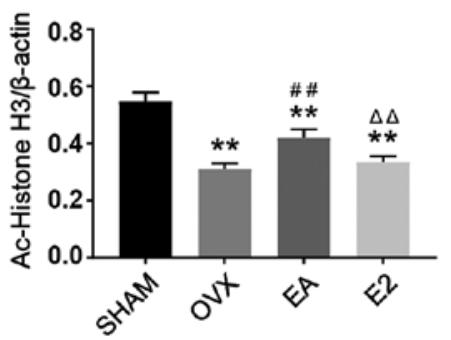

B

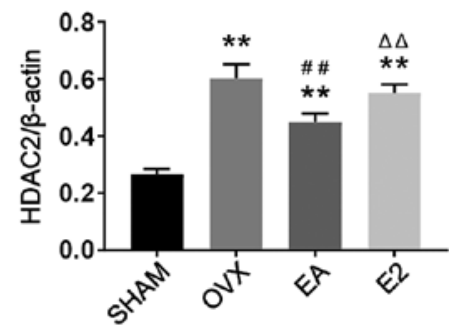

E

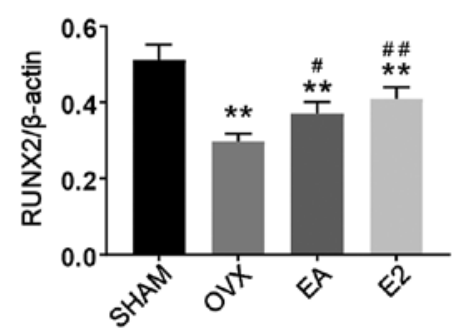

C

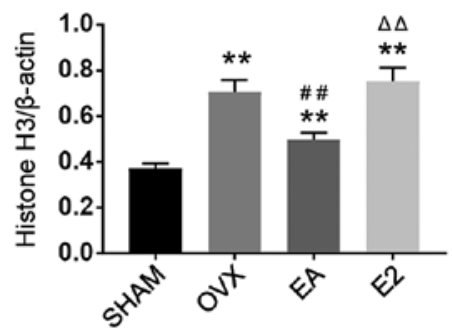

$\mathbf{F}$

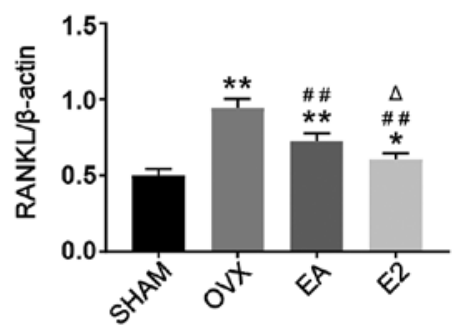

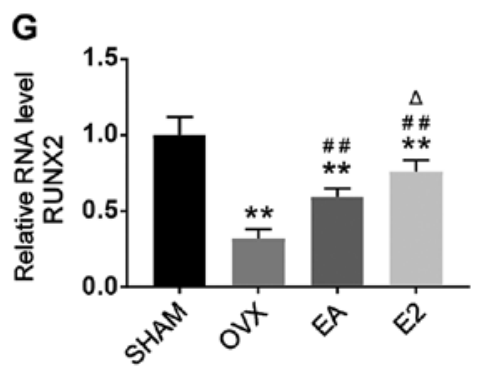

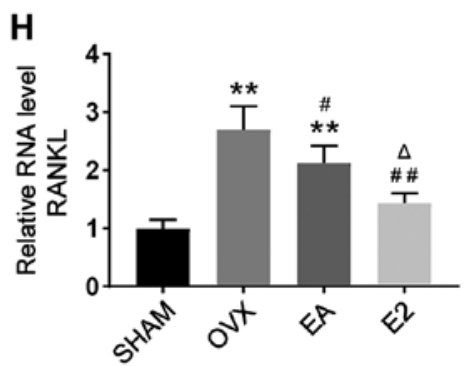

Figure 3. Comparison of protein and gene expression. (A) Representative western blot of HDAC2, histone H3, Ac-histone H3, RUNX2, RANKL and $\beta$-actin proteins in the SHAM, OVX model, EA and E2 treatment group; comparison of HDAC2 (B) histone H3 (C) Ac-histone H3 (D) RUNX2 (E) RANKL (F) protein expression in caput femoris of the four groups; comparison of (G) RUNX2 and (H) RANKL gene expression in caput femoris of the four groups. ${ }^{*} \mathrm{P}<0.05,{ }^{* *} \mathrm{P}<0.01$ vs. SHAM; ${ }^{\#} \mathrm{P}<0.05,{ }^{\# \#} \mathrm{P}<0.01$ vs. OVX; ${ }^{\circ} \mathrm{P}<0.05,{ }^{\Delta \Delta} \mathrm{P}<0.01$ vs. EA. SHAM, group given sham surgery leaving the ovaries intact; OVX, bilateral ovariectomy model group; EA, electroacupuncture treatment group; E2, 17 $\beta$-estradiol treatment control group; HDAC2, histone deacetylase 2; RUNX2, runt-related transcription factor 2 ; RANKL, receptor activator of nuclear factor- $\kappa \mathrm{B}$ ligand.

was downregulated by OVX, and was partially restored by treatment with either EA or E2. Compared with E2, EA was less effective in promoting RUNX2 mRNA expression. By contrast, RANKL mRNA was minimally expressed in the SHAM group in comparison with the OVX group. After 8 weeks of treatment with EA or E2, the mRNA expression of RANKL was suppressed in comparison with the OVX group, with E2 being more effective.

EA-mediated acetylation of histone $H 3$ regulates the differentiation of osteoblasts and osteoclasts. As shown in Fig. 4, Ac-histone H3, RUNX2 and ALP, which represent the activity of osteoblasts, were expressed throughout the caput femoris. Ac-histone $\mathrm{H} 3$ showed far more expression in the SHAM group than in the OVX group. After treatment with EA, the expression of Ac-histone H3 was restored, and there was no significant difference between the expressions of Ac-histone $\mathrm{H} 3$ in the $\mathrm{E} 2$ treatment group vs. the OVX group. RUNX2 and ALP were highly expressed in the SHAM group in comparison with the OVX group. After treatment with EA, the expression of RUNX2 and ALP were partially restored in the EA and E2 treatment groups. There were no significant differences between the EA and E2 treatment groups in the expression of RUNX2 and ALP, while the expression levels of RUNX2 and ALP in both groups were lower than those in the SHAM group. Correlation analysis revealed that Histone/RANKL and RANKL/TRACP were positive correlations, while the correlation of Histone/RANKL was weaker than that of RANKL/TRACP. This may also be related to the inability of E2 to regulate histone acetylation.

As shown in Fig. 5, histone H3, RANKL and TRACP, which reflect the activity of osteoclasts, were expressed throughout the caput femoris. Histone H3 was minimally expressed in the SHAM group as compared with the OVX group. After treatment with $\mathrm{EA}$, histone $\mathrm{H} 3$ was downregulated relative to that in the OVX group. After administration of E2, histone $\mathrm{H} 3$ levels were similar to those in the OVX group. As expected, based on the elevation of histone $\mathrm{H} 3$ after OVX, the levels of RANKL and TRACP, which are derepressed by histone H3, also increased after OVX. Treatment with EA or E2 mitigated these increases in RANKL and TRACP, and treatment with E2 was more effective than EA treatment. Correlation analysis 
A

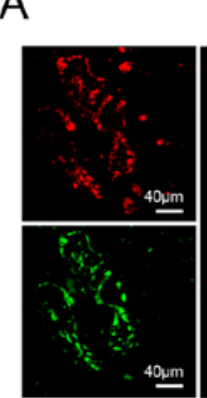

SHAM

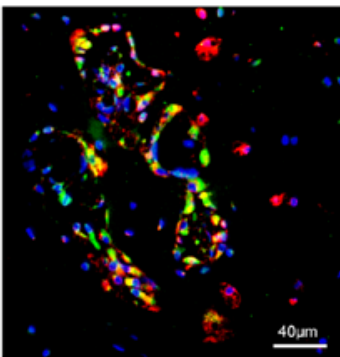

EA
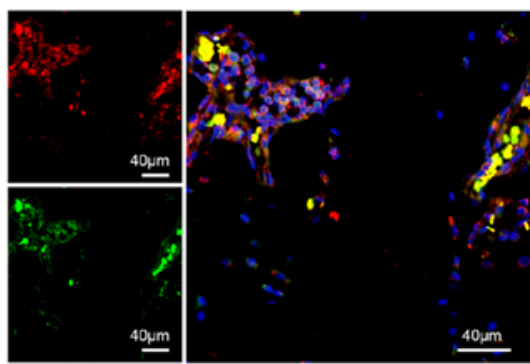

Ac-histone/ RUNX2/DAPI
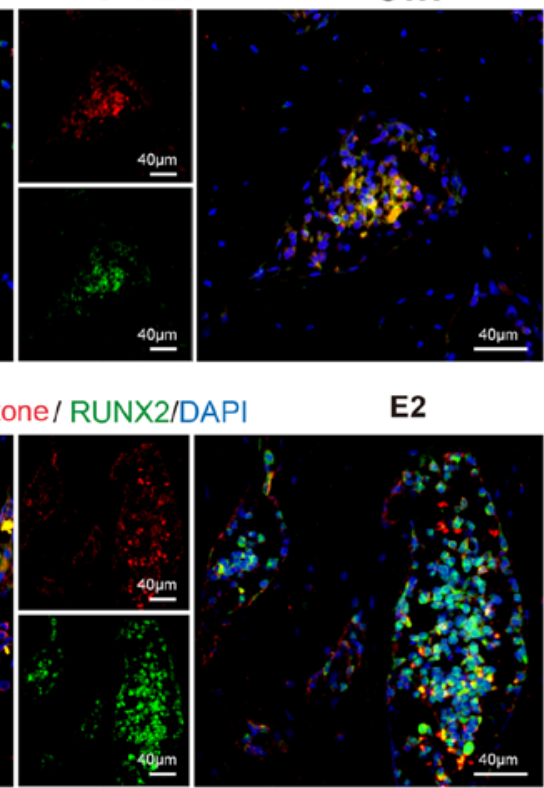

E2

B

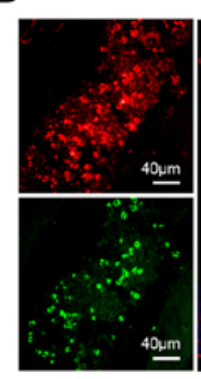

SHAM

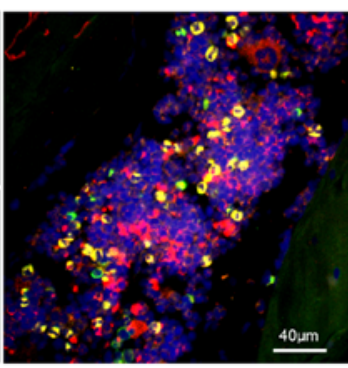

RUNX2 / ALP/DAPI
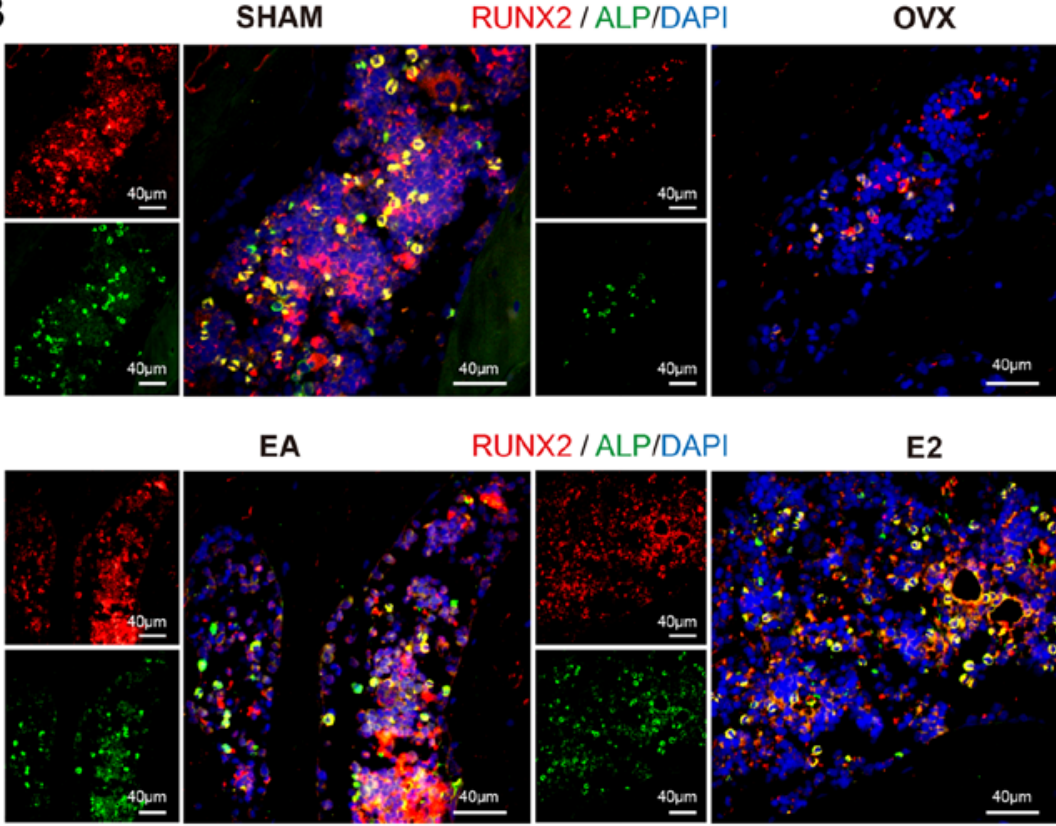

EA

RUNX2 / ALP/DAPI
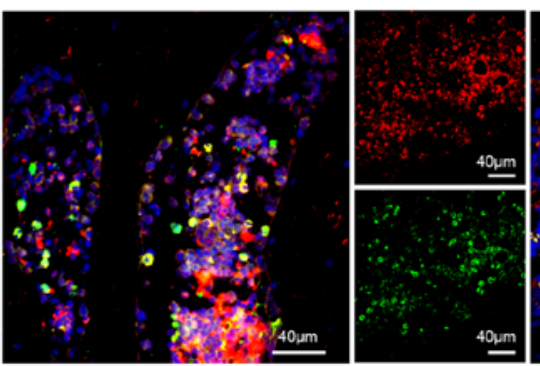

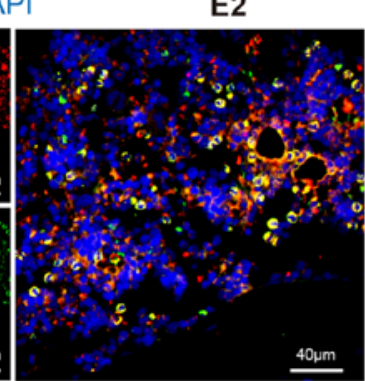

C
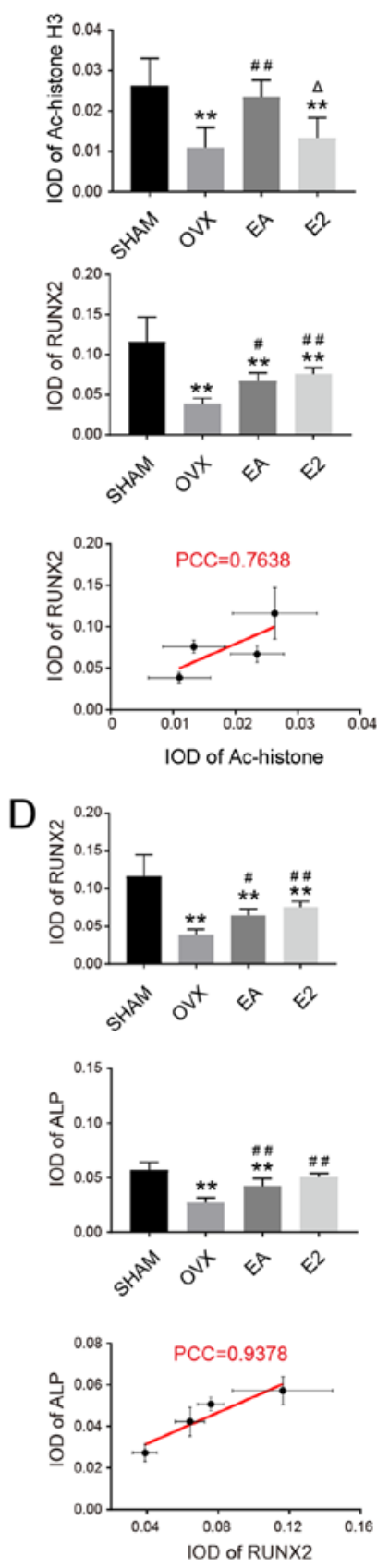

Figure 4. Representative double-labeled immunofluorescence images (A) Ac-histone H3 (red)/RUNX2 (green) and nuclei (blue). (B) RUNX2 (red)/ALP (green) and nuclei (blue). IOD from (C) Ac-histone H3/RUNX2 and (D) RUNX2/ALP. ${ }^{* *} \mathrm{P}<0.01$ vs. SHAM; ${ }^{*} \mathrm{P}<0.05,{ }^{\# \#} \mathrm{P}<0.01$ vs. OVX; ${ }^{\mathrm{P}} \mathrm{P}<0.05$ vs. EA. SHAM, group given sham surgery leaving the ovaries intact; OVX, bilateral ovariectomy model group; EA, electroacupuncture treatment group; E2, 17 $\beta$-estradiol treatment control group; PCC, Pearson correlation coefficient; IOD, integrated option density; Ac, acetylated; RUNX2, runt-related transcription factor 2; ALP, alkaline phosphatase.

revealed that Ac-Histone/RUNX2, RUNX2/ALP had a positive correlation, and the Histone/RUNX2 correlation was weaker than the RUNX2/ALP correlation. This may be related to the inability of E2 to regulate histone acetylation.

\section{Discussion}

Estrogen deficiency results in enhanced bone resorption accompanied by impaired bone formation, which are the main factors that affect BMD in patients with PMO. In the process of bone cell differentiation, estrogen is involved in the formation of osteoblasts and osteoclasts. RUNX2 is a transcription factor that determines the osteoblast lineage from mesenchymal stem cells via canonical Wnt signaling by blocking the inhibition of chondrocyte differentiation (22). Estrogen and selective estrogen receptor modulators induce RUNX2 mRNA expression and enhanced $R U N X 2$ promoter activity and play an important role in osteoblast differentiation $(23,24)$. RANKL, a type II transmembrane protein of the tumor necrosis factor superfamily, is mainly produced by osteoblastic lineage cells (mesenchymal stem cells, osteoblasts and osteocytes), and transduces its signal by binding the receptor RANK on the 
A

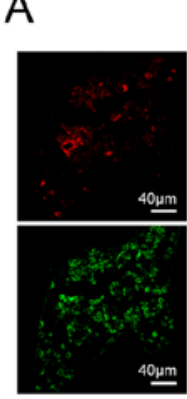

\section{SHAM}

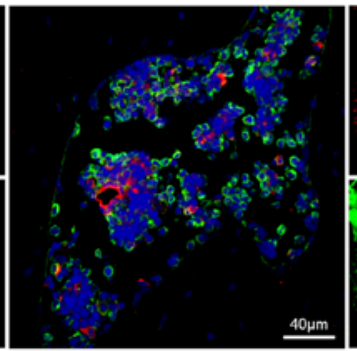

EA

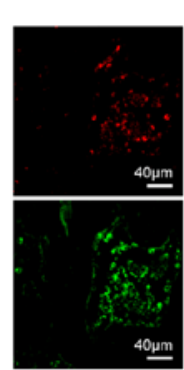

EA

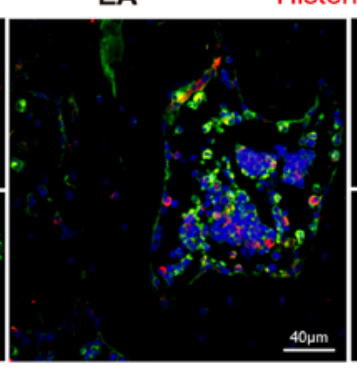

stone/ RANKL/DAP
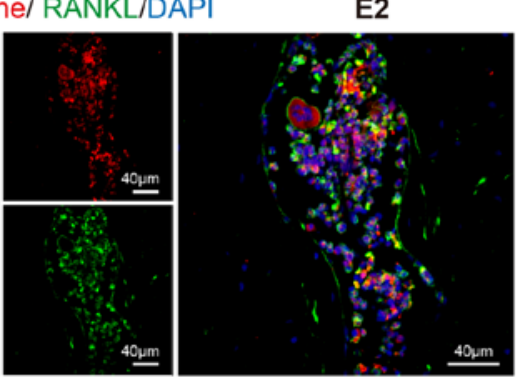

B

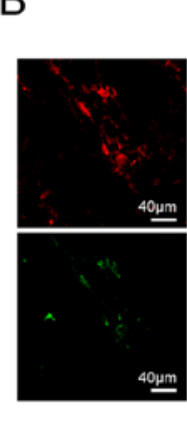

SHAM

RANKL/ TRACP/DAPI
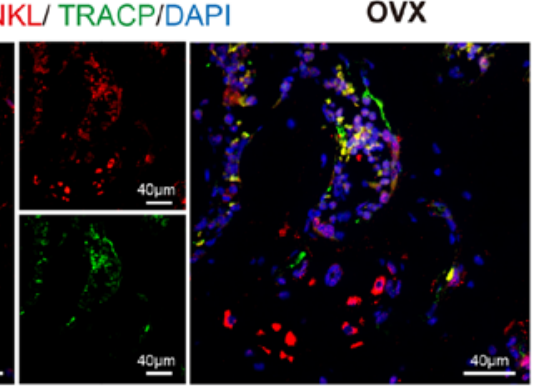

RANKL/ TRACP/DAPI

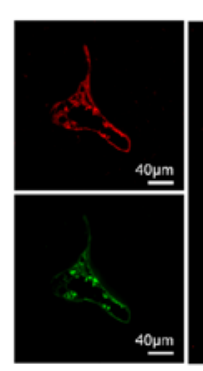

EA
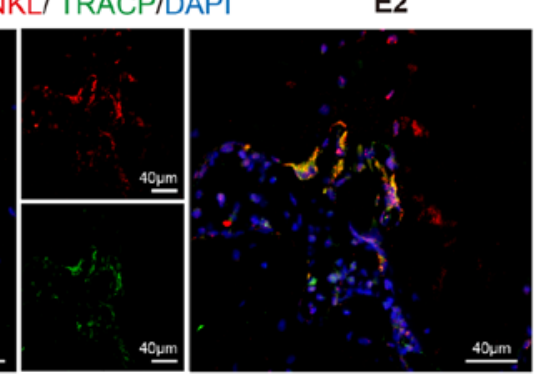
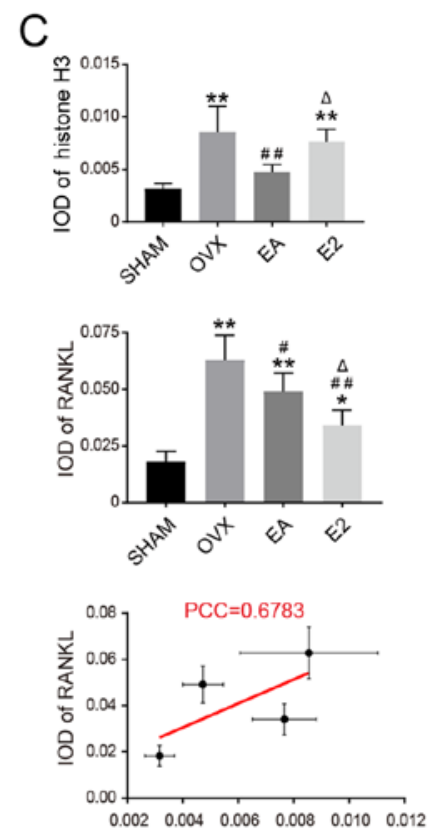

$1 \mathrm{OD}$ of histone $\mathrm{H} 3$

D
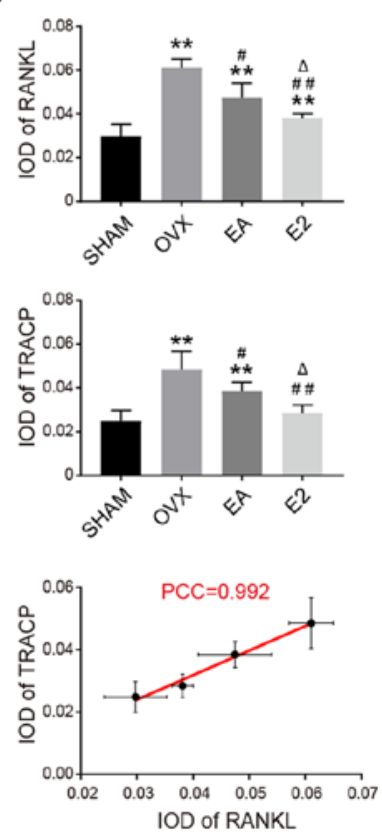

Figure 5. Representative double-labeled immunofluorescence images of (A) Histone H3 (red)/RANKL (green) and nuclei (blue). (B) RANKL (red)/TRACP (green) and nuclei (blue). IOD from (C) Histone H3/RANKL and (D) RANKL/TRACP. ${ }^{*} \mathrm{P}<0.05,{ }^{* *} \mathrm{P}<0.01$ vs. SHAM; ${ }^{*} \mathrm{P}<0.05,{ }^{\# \#} \mathrm{P}<0.01$ vs. OVX; ${ }^{\wedge} \mathrm{P}<0.05$ vs. EA. SHAM, group given sham surgery leaving the ovaries intact; OVX, bilateral ovariectomy model group; EA, electroacupuncture treatment group; E2, $17 \beta$-estradiol treatment control group; PCC, Pearson correlation coefficient; IOD, integrated option density; RANKL, receptor activator of nuclear factor- $\kappa B$ ligand; TRACP, tartrate-resistant acid phosphatase.

membrane of osteoclasts (25). This binding activates nuclear factor of activated T-cells, cytoplasmic 1, the master regulator of osteoclastogenesis, which induces the process of osteoclast differentiation from monocyte/macrophage lineage cells (10). Estrogen inhibits osteoporosis by promoting osteoclastic apoptosis and suppressing osteoclastogenesis (26). A potential mechanism by which estrogen has these effects may be related to the inhibitory effect of RANKL-induced osteoclastic differentiation by increasing the expression of the transient receptor potential cation channel subfamily $\mathrm{V}$ member 5 channel (27). In addition, estrogen can antagonize RUNX2-mediated osteoblast-driven osteoclastogenesis by regulating RANKL membrane association (9). Based on these effects, hormone replacement therapy (HRT) is still considered a first-line choice for the prevention of bone loss and fracture in the early postmenopausal period (28), and HRT was included as a positive control in our study. However, several side effects, including increased risk of endometrial carcinoma, breast cancer and thromboembolism, limit the clinical application of HRT (29).

According to the theory of epigenetics, the regulation of downstream gene expression by regulating acetylation of 


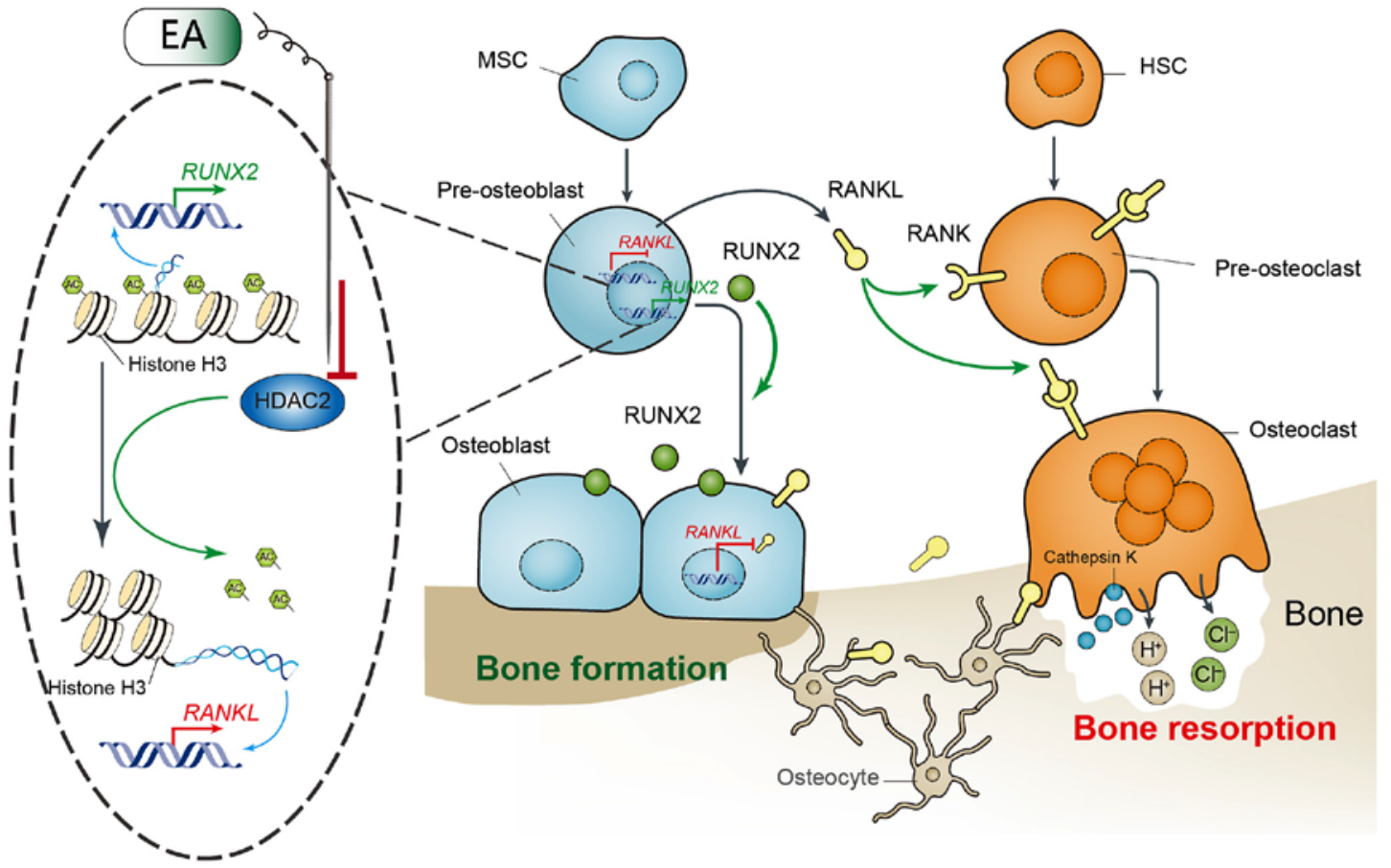

Figure 6. Schematic of potential mechanisms by which EA could mediate the acetylation of histone H3 and alter the differentiation of osteoblasts and osteoclasts. Ac, acetylated; EA, electroacupuncture; RANK, receptor activator of nuclear factor- $\kappa B$; RANKL, RANK ligand; RUNX2, runt-related transcription factor 2; MSC, mesenchymal stem cell; $\mathrm{HSC}$, hematopoietic stem cell; $\mathrm{H}^{+}$, hydrogen ions; $\mathrm{Cl}^{-}$, chloride ions.

histones is characterized by effects on multiple gene targets (13). Similarly, mechanisms of acupuncture to prevent and treat diseases are related to the regulation of various signaling pathways and molecules $(30,31)$, both these fields are characterized by pleotropic effects. Recently, acupuncture has been shown to regulate the hypothalamic-pituitary-ovarian axis (HPOA) in rats (32) and postmenopausal women (33), a finding which was widely applied in treatment of endocrine diseases (34). EA, characterized by sustained stimulation and the stability and controllability of its parameters, has been applied extensively in research and in clinical practice, including to HPOA dysfunction (35). When applied to improving osteoporosis, acupuncture can prevent bone loss and improve bone morphology by regulating osteoblast/osteoclast differentiation (36), and this effect was also observed in the present study. A possible mechanism may be through the regulation of the RANKL/RANK and Wnt/ $\beta$-catenin signaling pathways, which are known to suppress osteoclastogenesis (11). In addition, acupuncture also regulates downstream molecules including urine deoxypyridinoline, serum bone specific alkaline phosphatase (21), cathepsin K (37), TRACP and osteoprotegerin (36) in rat models of osteoporosis. These published studies have mainly explored the mechanism underlying acupuncture with respect to one or more cytokines or signal pathways related to osteoclastogenesis or osteoblastogenesis. The results of these studies fully illustrate the multi-target effect of acupuncture in improving osteoporosis. Nevertheless, whether this multi-target effect is related to histone acetylation levels has not been deeply studied. The underlying mechanisms of how acupuncture regulates these signaling pathways and molecules may be related to the modification of histones, which merits further study (38).

In the present study, the E2 group served as the positive treatment control, which was used to assess the efficacy of
EA and investigate the underlying mechanism. It was found that both EA and E2 improved the trabecular BMD and bone morphology of OVX-induced osteoporotic rats. However, neither EA nor E2 affected the Tb.Th of caput femoris. In addition, EA and E2 showed comparable improvements in the morphology of the trabecular bone. These results demonstrated the effectiveness of hormone replacement therapy and suggested that applying EA alone can achieve similar effects. The study also found that the levels of HDAC2 in caputs femoris were increased after OVX, which induced increased expression of histone $\mathrm{H} 3$ and lower expression of Ac-histone H3. Administration of EA mitigated these OVX-induced HDAC2 increases. Enhancement of histone acetylation levels were correlated with the expected changes in RUNX2 and RANKL, presumably via changes in the three-dimensional structure of chromatin. Correspondingly, RUNX2 protein expression was upregulated and RANKL protein expression was downregulated following EA treatment. In the results of IF, ALP-labelled osteoblasts were decreased in caput femoris sections after OVX. These decreases were mitigated by EA or E2 treatment, and RUNX2 protein expression changes were similar to those of ALP in the caput femoris tissues. As expected, the changes in RANKL/TRACP labelling in caput femoris were generally opposite to those of RUNX2/ALP. Consistent with the results of IF, the bone metabolism biochemical markers including TRACP, ALP and E2 in serum were also changed. These results suggested that the alterations in the protein and gene expression of RUNX2 and RANKL affected the differentiation of osteoblasts and osteoclasts, which regulated BMD and trabecular morphology. By contrast, administration of E2 in OVX rats did not change the protein expression of HDAC2 and did not affect the level of histone acetylation. However, the protein and gene expression of RUNX2 and RANKL were 
affected by E2, which indicates that the underlying mechanisms by which EA and E2 treat PMO were different.

In conclusion, the present study demonstrated that EA suppressed bone loss and improved trabecular morphology in rats with OVX-induced osteoporosis. Furthermore, a potential mechanism was identified through EA-induced epigenetic changes which may be linked to the observed effects. EA suppressed the activity of HDAC2 and increased the acetylation of histone $\mathrm{H} 3$ in caput femoris, which increased the gene and protein expression of RUNX2 and decreased those of RANKL. The alternations of expression of RUNX2 and RANKL readjusted the differentiation of osteoblasts and osteoclasts (Fig. 6). The mechanism of action from treatment with EA was different from that of treatment with E2, in that E2 did not modulate the activity of HDAC2. Despite these findings, this study has limitations. Chromatin immunoprecipitation (CHIP)-PCR was not used to detect the relationship between histone $\mathrm{H} 3$ and the expression of the RUNX2/RANKL genes, and the possible downstream genetics affected by EA-induced histone $\mathrm{H} 3$ acetylation need further investigation through CHIP-seq. Thus, the potential mechanisms underlying how EA improves osteoporosis will be investigated in further systematic studies that could provide additional evidence for the application of acupuncture in preventing and treating osteoporosis. Furthermore, based on the multi-target characteristics of the effects of acupuncture and epigenetic regulation, the results of this study show some possible directions for further research on the mechanisms underlying acupuncture and provide more evidence for application of acupuncture.

\section{Acknowledgements}

Not applicable.

\section{Funding}

This work was supported by Natural Science Foundation of Hubei Province (grant no. 2017CFB787) and the Fundamental Research Funds for the Central Universities (grant no. 2042018kf0097).

\section{Availability of data and materials}

All data generated or analyzed during this study are included in this published article.

\section{Authors' contributions}

JT and QS conceived the project. QS and RL designed the experiments. QS, YS and YH performed the experiments. ZP analyzed the data. QS and JT wrote the manuscript. All authors read and approved the final manuscript.

\section{Ethics approval and consent to participate}

All animal procedures were performed according to the Declaration of Helsinki (European Union guidelines on use of animals in scientific experiments) and Animal Research: Reporting of In Vivo Experiments (ARRIVE) guidelines. The study was approved by the Institutional Animal Care and Use
Committee of Wuhan University, Wuhan, Hubei, P.R. China (no. 2018122).

\section{Patient consent for publication}

Not applicable.

\section{Competing interests}

The authors declare that they have no competing interests.

\section{References}

1. Eastell R, O'Neill TW, Hofbauer LC, Langdahl B, Reid IR, Gold DT and Cummings SR: Postmenopausal osteoporosis. Nat Rev Dis Primers 2: 16069, 2016.

2. Manolagas SC, O'Brien CA and Almeida M: The role of estrogen and androgen receptors in bone health and disease. Nat Rev Endocrinol 9: 699-712, 2013.

3. Eastell R, Rosen CJ, Black DM, Cheung AM, Murad MH and Shoback D: Pharmacological management of osteoporosis in postmenopausal women: An endocrine society" clinical practice guideline. J Clin Endocrinol Metab 104: 1595-1622, 2019.

4. Manson JE, Chlebowski RT, Stefanick ML, Aragaki AK, Rossouw JE, Prentice RL, Anderson G, Howard BV, Thomson CA, LaCroix AZ, et al: Menopausal hormone therapy and health outcomes during the intervention and extended poststopping phases of the women's health initiative randomized trials. JAMA 310: 1353-1368, 2013.

5. Axelsson KF, Wallander M, Johansson H, Lundh D and Lorentzon M: Hip fracture risk and safety with alendronate treatment in the oldest-old. J Intern Med 282: 546-559, 2017.

6. Hiligsmann M, Williams SA, Fitzpatrick LA, Silverman SS Weiss R and Reginster JY: Cost-effectiveness of sequential treatment with abaloparatide vs. teriparatide for United States women at increased risk of fracture. Semin Arthritis Rheum 49: 184-196, 2019.

7. Pan H, Jin R, Li M, Liu Z, Xie Q and Wang P: The effectiveness of acupuncture for osteoporosis: A systematic review and meta-analysis. Am J Chin Med 46: 489-513, 2018.

8. Fan H, Ji F, Lin Y, Zhang M, Qin W, Zhou Q and Wu Q: Electroacupuncture stimulation at CV4 prevents ovariectomy-induced osteoporosis in rats via Wnt- $\beta$-catenin signaling. Mol Med Rep 13: 2485-2491, 2016.

9. Martin A, Xiong J, Koromila T, Ji JS, Chang S, Song YS, Miller JL, Han CY, Kostenuik P, Krum SA, et al: Estrogens antagonize RUNX2-mediated osteoblast-driven osteoclastogenesis through regulating RANKL membrane association. Bone 75: 96-104, 2015.

10. Ono $\mathrm{T}$ and Nakashima T: Recent advances in osteoclast biology. Histochem Cell Biol 149: 325-341, 2018.

11. Zheng $X, \mathrm{WuG}, \mathrm{Nie} Y$ and Lin $Y$ :Electroacupuncture at the governor vessel and bladder meridian acupoints improves postmenopausal osteoporosis through osteoprotegerin/RANKL/RANK and Wnt/ $\beta$-catenin signaling pathways. Exp Ther Med 10: 541-548, 2015.

12. Shen Y, Wei W and Zhou DX: Histone acetylation enzymes coordinate metabolism and gene expression. Trends Plant Sci 20: 614-621, 2015.

13. Gräff J and Tsai LH: Histone acetylation: Molecular mnemonics on the chromatin. Nat Rev Neurosci 14: 97-111, 2013.

14. Marini F, Cianferotti L and Brandi ML: Epigenetic mechanisms in bone biology and osteoporosis: Can they drive therapeutic choices? Int J Mol Sci 17: 1329, 2016.

15. Dou C, Li N, Ding N, Liu C, Yang X, Kang F, Cao Z, Quan H, Hou $\mathrm{T}, \mathrm{Xu} \mathrm{J}$ and Dong S: HDAC2 regulates FoxO1 during RANKL-induced osteoclastogenesis. Am J Physiol Cell Physiol 310: C780-C787, 2016.

16. Queirolo V, Galli D, Masselli E, Borzi RM, Martini S, Vitale F, Gobbi G, Carubbi C and Mirandola P: PKCepsilon is a regulator of hypertrophic differentiation of chondrocytes in osteoarthritis. Osteoarthritis Cartilage 24: 1451-1460, 2016.

17. Li J, Wu S, Tang H, Huang W, Wang L, Zhou H, Zhou M, Wang H and Li J: Long-term effects of acupuncture treatment on airway smooth muscle in a rat model of smoke-induced chronic obstructive pulmonary disease. Acupunct Med 34: 107-113, 2016. 
18. Tu WZ, Cheng RD, Cheng B, Lu J, Cao F, Lin HY, Jiang YX, Wang JZ, Chen $\mathrm{H}$ and Jiang SH: Analgesic effect of electroacupuncture on chronic neuropathic pain mediated by $\mathrm{P} 2 \mathrm{X} 3$ receptors in rat dorsal root ganglion neurons. Neurochem Int 60: 379-386, 2012

19. Yin CS, Jeong HS, Park HJ, Baik Y, Yoon MH, Choi CB and Koh HG: A proposed transpositional acupoint system in a mouse and rat model. Res Vet Sci 84: 159-165, 2008.

20. Shu Q, Chen L, Wu S, Li J, Liu J, Xiao L, Chen R and Liang F: Acupuncture targeting SIRT1 in the hypothalamic arcuate nucleus can improve obesity in high-fat-diet-induced rats with insulin resistance via an anorectic effect. Obes Facts 13: 40-57, 2020.

21. Wang HD, Chen Z, Inoue I, Fu SJ, Shi XL, Tang L, Zhang FZ, Jiang Y and Jiang H: Effects of electroacupuncture at GB points on markers of osteoporosis and bodyweight in ovariectomised rats. Acupunct Med 33: 465-471, 2015.

22. Komori T: Runx2, an inducer of osteoblast and chondrocyte differentiation. Histochem Cell Biol 149: 313-323, 2018.

23. Amzaleg Y,JiJ,KittivanichkulD,Törnqvist AE,WindahlS,SabagE Khalid AB, Sternberg H, West M, Katzenellenbogen JA, et al: Estrogens and selective estrogen receptor modulators differentially antagonize Runx2 in ST2 mesenchymal progenitor cells. J Steroid Biochem Mol Biol 183: 10-17, 2018

24. Wang Y, Lu Y, Li Z, Zhou Y, Gu Y, Pang X, Wu J, Gobin R and $\mathrm{Yu}$ J: Oestrogen receptor $\alpha$ regulates the odonto/osteogenic differentiation of stem cells from apical papilla via ERK and JNK MAPK pathways. Cell Prolif 51: e12485, 2018.

25. Sobacchi C, Menale $C$ and Villa A: The RANKL-RANK Axis: A bone to thymus round trip. Front Immunol 10: 629, 2019.

26. Xiong Q, Tang P, Gao Y, Zhang L and Ge W: Proteomic analysis of estrogen-mediated signal transduction in osteoclasts formation. Biomed Res Int 2015: 596789, 2015.

27. Chen F, Ouyang Y, Ye T, Ni B and Chen A: Estrogen inhibits RANKL-induced osteoclastic differentiation by increasing the expression of TRPV5 channel. J Cell Biochem 115: 651-658, 2014.

28. Tella SH and Gallagher JC: Prevention and treatment of postmenopausal osteoporosis. J Steroid Biochem Mol Biol 142: $155-170,2014$

29. Gambacciani $M$ and Levancini $M$ : Hormone replacement therapy and the prevention of postmenopausal osteoporosis. Prz Menopauzalny 13: 213-220, 2014.
30. Wang Y, Jiang H, Meng H, Li J, Yang X, Zhao B, Sun Y and Bao T: Antidepressant mechanism research of acupuncture: Insights from a genome-wide transcriptome analysis of frontal cortex in rats with chronic restraint stress. Evid Based Complement Alternat Med 2017: 1676808, 2017.

31. Zhang A, Yan G, Sun H, Cheng W, Meng X, Liu L, Xie N and Wang X: Deciphering the biological effects of acupuncture treatment modulating multiple metabolism pathways. Sci Rep 6: 19942, 2016.

32. Fu H, Sun J, Tan Y, Zhou H, Xu W, Zhou J, Chen D, Zhang C, Zhu X, Zhang Y, et al: Effects of acupuncture on the levels of serum estradiol and pituitary estrogen receptor beta in a rat model of induced super ovulation. Life Sci 197: 109-113, 2018.

33. Chen GZ, Xu YX, Zhang JW, Liu SH and Guo ZY: Effect of acupoint catgut-embedding on the quality of life, reproductive endocrine and bone metabolism of postmenopausal women. Chin J Integr Med 16: 498-503, 2010.

34. Deng YZ, Li LB, Xu LG, Zhou D, Wei LJ and Liu Y: Acupuncture in endocrine disorders: A critical appraisal. J Biol Regul Homeost Agents 30: 1035-1040, 2016.

35. Zhao H, Tian ZZ and Chen BY: An important role of corticotropin-releasing hormone in electroacupuncture normalizing the subnormal function of hypothalamus-pituitary-ovary axis in ovariectomized rats. Neurosci Lett 349: 25-28, 2003.

36. Zheng X, Nie Y, Sun C, Wu G, Cai Q, Huang S and Lin Y: Long-term electroacupuncture stimulation prevents osteoporosis in ovariectomised osteopaenic rats through multiple signalling pathways. Acupunct Med 36: 176-182, 2018.

37. Zhou J, Li X, Liao Y, Feng W and Guo X: Effects of electroacupuncture on bone mass and cathepsin $\mathrm{K}$ expression in ovariectomised rats. Acupunct Med 32: 478-485, 2014.

38. Mak JC: Acupuncture in osteoporosis: More evidence is needed. Acupunct Med 33: 440-441, 2015.

c) (i) $\Theta$ This work is licensed under a Creative Commons Attribution-NonCommercial-NoDerivatives 4.0 International (CC BY-NC-ND 4.0) License. 